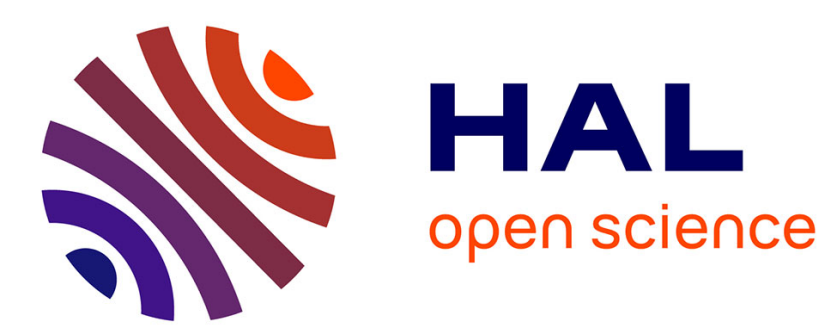

\title{
Color and Figure-Ground: From Signals to Qualia
}

Birgitta Dresp, Adam Reeves

\section{To cite this version:}

Birgitta Dresp, Adam Reeves. Color and Figure-Ground: From Signals to Qualia. S. Magnussen, M Greenlee, J. Werner, A. Geremek. Perception Beyond Gestalt: Progress in Vision Research, Routledge/Taylor \& Francis, pp.159-171, 2013. hal-01053969

\section{HAL Id: hal-01053969 \\ https://hal.science/hal-01053969}

Submitted on 4 Aug 2014

HAL is a multi-disciplinary open access archive for the deposit and dissemination of scientific research documents, whether they are published or not. The documents may come from teaching and research institutions in France or abroad, or from public or private research centers.
L'archive ouverte pluridisciplinaire HAL, est destinée au dépôt et à la diffusion de documents scientifiques de niveau recherche, publiés ou non, émanant des établissements d'enseignement et de recherche français ou étrangers, des laboratoires publics ou privés. 


\title{
12 Color and figure-ground
}

\author{
From signals to qualia
}

\author{
Birgitta Dresp-Langley and Adam Reeves
}

\section{Introduction}

The laws which predict how the perceptual quality of figure-ground can be extracted from the most elementary visual signals were discovered by the Gestaltists, and form an essential part of their movement (see especially Metzger, 1930, and Wertheimer, 1923 translated and re-edited by Lothar Spillmann, 2009 and 2012, respectively). Distinguishing figure from ground is a prerequisite for perception of both form and space (the relative positions, trajectories, and distances of objects in the visual field). The human brain has an astonishing capacity for selecting and combining a few critical visual signals, through complex mechanisms, to represent form and space accurately. The phenomenon of figureground in its most elementary form may be seen in the famous Ehrenstein illusion (Ehrenstein, 1941, studied extensively by Spillmann and colleagues, see DrespLangley, 2009, for a general review). In this illusion, four thin lines form a cross configuration with an empty centre (as in Figure 12.1).

If the converging lines are dark on a lighter background, the centre appears to fill in with a disk shape that appears even lighter than the background. With white inducing lines on a darker background, the centre appears to fill in with an even darker disk. These, typically asymmetrical, perceptual sensations of brightness or darkness enhancement are deemed illusory because they have no physical origin given that the luminance at the centre of the figure is identical to the background luminance. The illusion illustrates simultaneous contrast (Spillmann, 1977; Spillmann et al., 1984), where surfaces surrounded by regions of opposite contrast polarity appear to brighten or to darken, but its most salient phenomenal attribute is the apparent figural enhancement at the centre of the configuration, induced by the four converging lines (Spillmann et al., 1976). The figural enhancement, an opaque disk placed on what could be a cross lying underneath, involves both structural depth and figure-ground (Spillmann and Dresp, 1995; Watanabe et al., 1995), as the illusory centre appears to lie in a distinct depth plane from the background. The original Ehrenstein illusion results from orientation and luminance signals. With colored inducers, the resulting figure-ground percept suggests neon-color spreading (for a review see Spillmann and Dresp, 1995). Color signals can generate strong and self-sufficient cues to figure-ground, as for example in the 


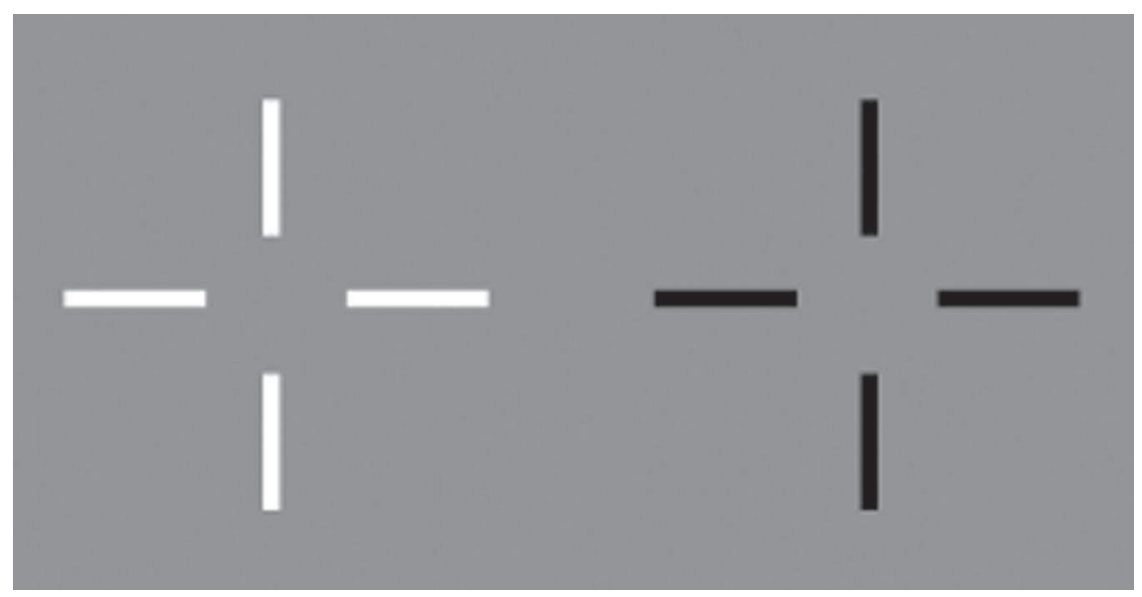

Figure 12.1 Examples of the Ehrenstein illusion, which was extensively studied by Spillmann and his colleagues. A shape percept is induced by dark or bright converging lines, where a disk lying in front of the display is perceived.

watercolor illusion discovered by Pinna and colleagues (2001; see also Pinna and Reeves, 2006 and von der Heydt and Pierson, 2006), and in the related van Tuijl Illusion (1975). Interestingly, color signals associated or not with a luminance contrast may either strengthen or override geometric cues to figure-ground (Dresp et al., 2002; Guibal and Dresp, 2004). In these experiments, perceptually transparent or opaque crosses were presented, and observers judged whether the horizontal or vertical component appeared nearer.

\section{Perceptual space from planar objects: the role of luminance contrast and geometric cues}

Planar geometry provides powerful cues to depth, as shown in Figure 12.2 (see Color plate XX in the Color plate section). Such cues include the relative height of stimuli in the visual field (top), structural interposition without occlusion suggesting transparent overlapping forms (middle), and cues to partial occlusion suggesting an opaque occluding object in front of another (bottom).

In experiments by Guibal and Dresp (2004), the brighter and darker levels of the colors red and green were equiluminant with regard to each other, their luminance contrast with the grey background varied. The luminance contrast of a given color, supported by one of the three geometric cues, determines the probability of a given figural element to be perceived as nearer to the human observer, or as figure versus ground, as in Figure 12.3a. Response times for 'near' decrease with increasing luminance contrast (Figure 12.3b), suggesting that contrast increases salience. Interactions between luminance contrast and geometric cues, as shown here, generate variations in the relative visibility of parts, 
a)

Probability of figure part to appear "nearer" as a function

of its luminance contrast suported by one of three geometric cues

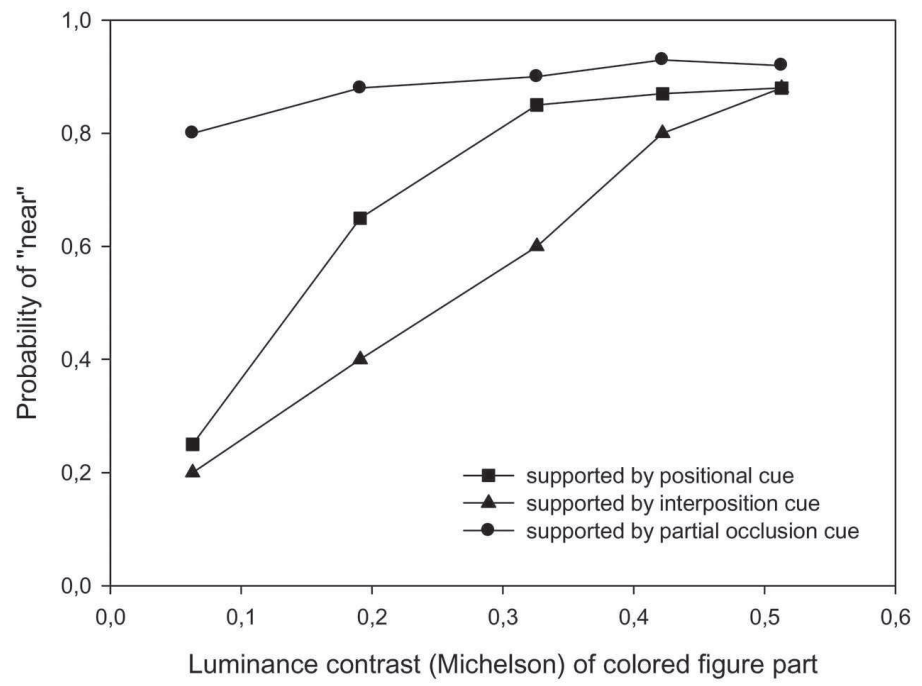

b)

Response latency as a function of the luminance contrast of the figure part appearing "nearer" supported by one of three geometric cues

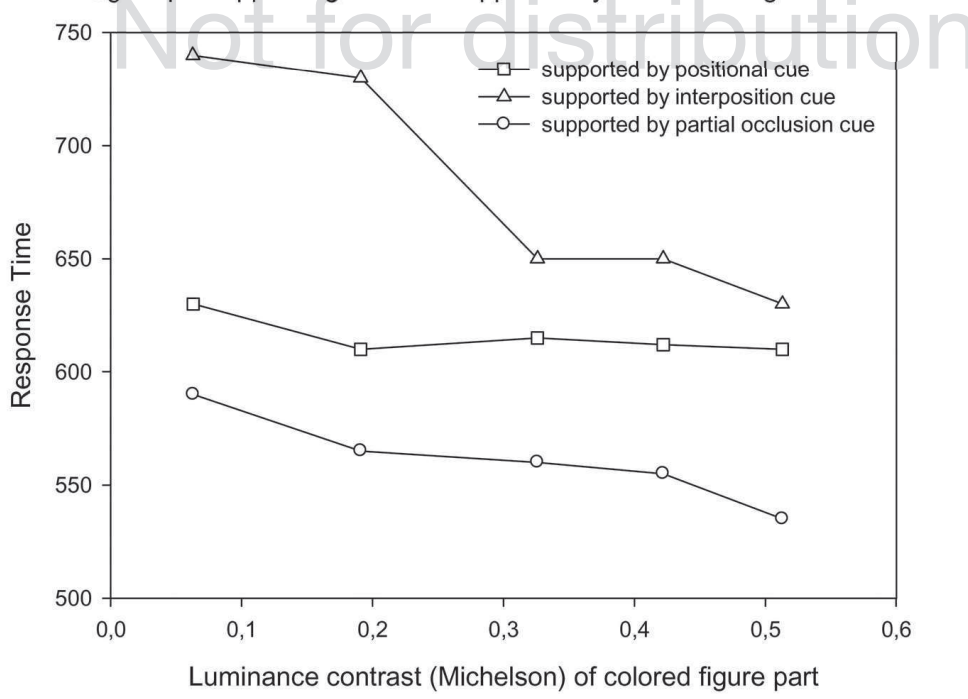

Figure 12.3 Response probabilities of 'near' (top) and response latencies (bottom) as a function of the luminance contrast (Michelson) of the selected visual part, combined with one of the three different geometric cues to figure-ground illustrated in Figure 12.2 (after Dresp et al., 2002). 


\section{Dresp-Langley, Reeves}

where partial occlusion is a strong pictorial cue to figure-ground and may support a weaker luminance contrast. Interposition cues lose out against spatial position and partial occlusion in determining the probability of 'near' at all but the highest contrast.

Why the relative visibility of a color determines pictorial depth is explained by the fact that contrast simulates the laws of aerial perspective (Ross, 1967). Schwartz and Sperling (1983), Egusa (1983), and Rohaly and Wilson (1999) showed that the perceived depth of a given region within the visual field is determined by the brightness of that region (as illustrated here in Figure 12.4). O'Shea and co-workers (1994) demonstrated, in binocular and monocular viewing, that the higher-contrast stimulus of a pair systematically appears nearer

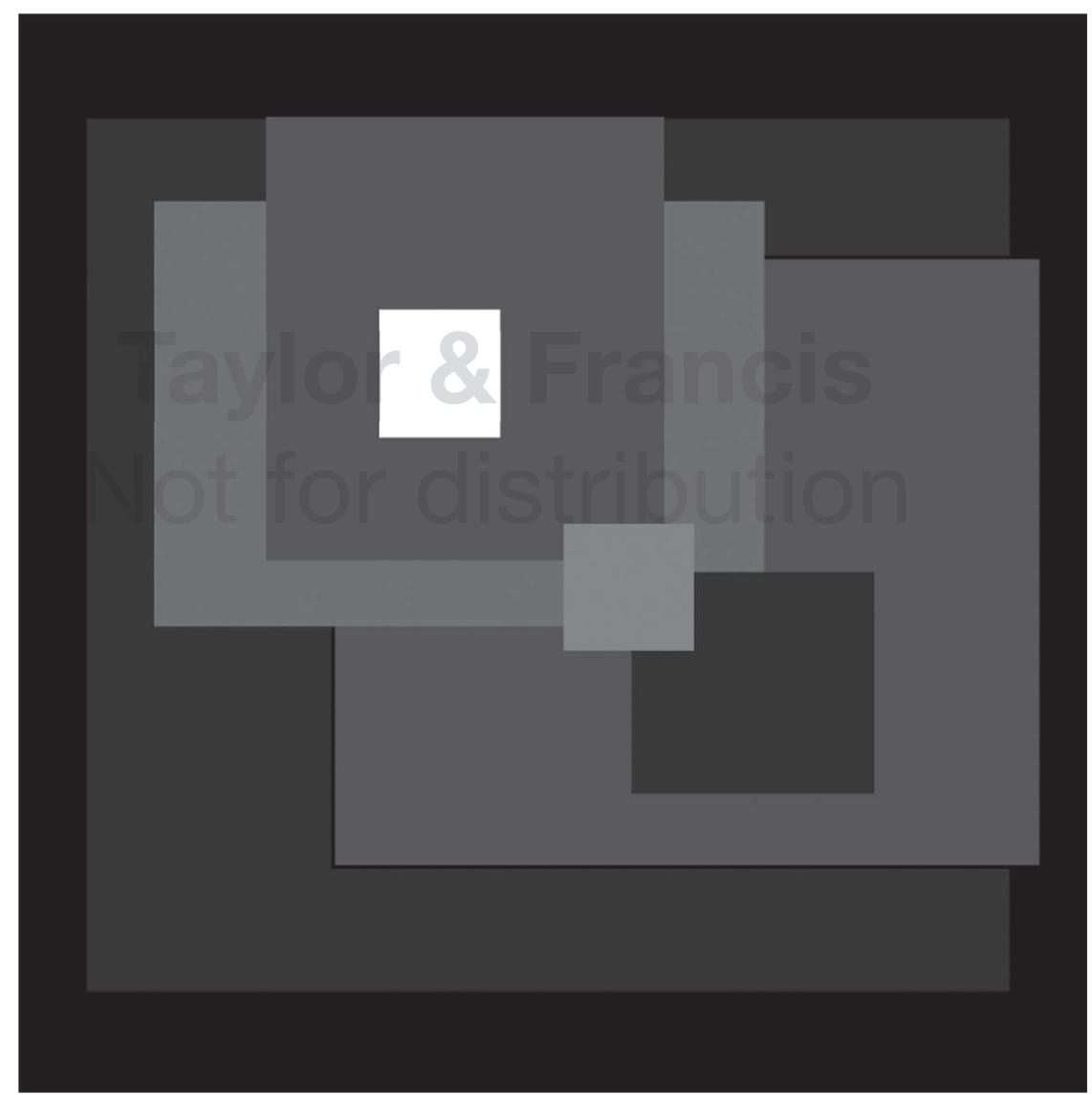

Figure 12.4 Objects with stronger luminance contrast in the plane tend to be seen as nearer to the observer than objects with weaker luminance contrast. Here, the small bright square with the highest contrast is likely to be seen as nearer than the small grey square even though the latter is supported by multiple cooperative interposition cues.

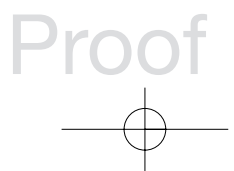


than the lower-contrast stimulus. Moreover, when a luminance cue was tested against a size cue for linear perspective, luminance prevailed over size. Schwartz and Sperling (1967) found that brighter stimuli had a stronger tendency to stand out in front even when perspective cues suggested the opposite. Luminance contrast determines which shape of a pair in a configuration will be seen as nearer (Dresp et al., 2002), and a sufficiently strong luminance cue may prevail over structural cues to depth such as interposition and partial occlusion.

\section{Surface effects from color}

Color has a particular status in perceptual organization (Oyama and Yamamura, 1967), partly because of the well-known phenomenon of color stereopsis (Brewster, 1851; Verhoeff, 1928). Color stereopsis refers to the effect of color contrast on the perceived depth of stimuli when they are viewed binocularly, through a convex lens or with the unaided eye, and when their luminance contrast does not vary. Experiments on color stereopsis have revealed a difference between long-wavelength stimuli such as red or yellow and short-wavelength stimuli such as blue or green when participants are asked to judge which color appears closer (Brewster, 1851). When viewed binocularly, with the aided or unaided eye, reds and yellows typically appear closer than blues or greens. Whether this suggests an intrinsically competitive nature of colors, predicted by their wavelength and possibly under the influence of other cues such as luminance contrast or stimulus geometry, remains to be clarified. Observations by Dengler and Nitschke (1993) have shown that changes in contrast at the borders of isoluminant orange and blue lines or squares may induce depth reversals. When these colors were lighter than the background, the long-wavelength color orange was seen in front of the shortwavelength color blue, but when the colors were darker than the background, blue was seen in front. The relative weight of the contribution of color stereopsis to processes of perceptual organization has remained unclear. When stereopsis is not at issue, color is still a critical factor, and the most valuable observations suggesting why this may be the case have been reported almost two centuries ago by the French chemist Michel-Eugène Chevreul.

\section{Chevreul's laws}

The idea that color is a powerful cue to figure-ground organization preceded the Gestalt movement and color psychophysics by almost half a century. In 1839, well before Katz published his observations on color experience (1911), and about 12 years before color stereopsis was discovered by Brewster (1851), Chevreul published his observations on the perceptual modifications produced by the mutual proximity of colors ( De la loi du contraste simultané des couleurs et de l'assortiment des objets colorés, Chevreul, 1839). He defined what later has become known as simultaneous color contrast (e.g. Beck, 1966; De Weert and Spillmann, 1995; Gerrits and Vendrik, 1970; Heinemann, 1955; Shapley and Reid, 1985). Observing how colored surfaces placed side by side or surrounding each other 


\section{Dresp-Langley, Reeves}

change in appearance according to which color is put next to which other, Chevreul suggested how they needed to be displayed in space to produce specific effects on the perception of a human observer. His laws of color and contrast have provided valuable intuitions about the effect of color on perceptual organization, inspiring artists, architects, designers, and visual scientists ever since.

\section{Law of 'true' color}

Chevreul's laws are important with regard to perceptual qualities such as color saliency and perceptual organization. One such law is concerned with the conditions necessary for the perception of unblemished, true colors, predicting that a color presented on grey will truly appear to the observer as that particular color, producing no mutual interactions with the background that alter the appearance of either. This law is based on Chevreul's observation that all primary colors appear 'pure' and 'true' by the proximity of grey while black and white affect a color's brightness and the perception of its tone. Psychophysical data have shown since that placing a color near other so-called inducing colors changes the appearance in either brightness or tint of the test color often dramatically (e.g. Livitz et al., 2011). This phenomenon may be reflected by a contrast effect, where the perceived brightness or tone of the test color changes away from that of the inducing color, or by an assimilation effect, where the perceived brightness or tone of the test color changes toward that of the inducing color. Interaction of a similar kind occurs between achromatic stimuli of positive and negative contrast polarities, producing either contrast, where a bright surface makes an adjacent one look darker and a dark one makes an adjacent one look brighter, or assimilation, where a bright surface makes an adjacent one look brighter and a dark surface makes an adjacent one look darker (e.g. Beck, 1966; Hamada, 1985; Heinemann, 1955). Chevreul's law of true color has never been challenged by psychophysics, and mutual interactions where grey fields change the appearance of nearby or surrounding colors, or colors change the appearance of nearby or surrounding grey fields, have only very recently been investigated (Dresp-Langley and Reeves, 2012).

\section{Law of contrast}

A color will be seen as figure rather than as ground when the difference in luminance between the color and its background is the strongest. This was first predicted by Chevreul's law of contrast and, more than a century later, confirmed by the psychophysical studies referred to above, showing that surfaces with the stronger luminance contrast tend to be perceived as figure rather than as ground, or as nearer to the human observer than surfaces with the weaker luminance contrast. The effect of frames on the appearance of tones and their brightness, or 'the difference between the effect of a framed picture and the effect of that same picture when seen through an opening' was considered by Chevreul (1839) to be of crucial importance to figure-ground relations in the visual field of the human

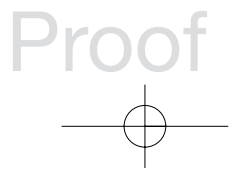


observer. What he called the 'contiguity of the frame' is claimed to alter any of the perceptual effects produced by any of his laws under conditions where no frame is present. This intuition that distinct object borders influence our perception is consistent with studies showing interactions between color appearance and the spatial profile of surface contours and geometric configuration (Devinck et al., 2006; De Weert and Spillmann, 1995; Dresp and Fischer, 2001; Pinna, 2008; 2011; Pinna and Reeves, 2006).

A compelling phenomenon revealing the simultaneous contrast effects of color patterns on achromatic backgrounds is shown in Figure 12.5 (see Color plate XX in the Color plate section). The colored squares, darker on one side of the display and brighter on the other, are placed on spatially separated grey fields of homogenous intensity and presented on a dark (black) screen. The colors' immediate grey background is systematically seen as brighter on one side. The induction can switch from contrast, where the grey field containing the darker inducers appears brighter, to assimilation, where the grey field containing the brighter inducers appears brighter. The effects strongly depend on the geometric configuration of the backgrounds. Surprisingly little is still known about the perceptual interactions between color and achromatic backgrounds. In complex configurations of color on grey, figure-ground percepts are not influenced by lighting conditions or visual adaptation levels (Dresp-Langley and Reeves, 2012). Simonet and Campbell (1990) reported effects of illumination levels on color stereopsis suggesting a contribution to figure-ground analysis at early stages of visual processing (see also Dengler and Nitschke, 1993). However, color stereopsis does not predict how the colors will interact with geometric cues in perceptual organization. Guibal and Dresp (2004) found that partial occlusion combined with the colors green or white produced weaker depth percepts and slower perceptual judgments than partial occlusion combined with the color red, regardless of background intensity. At the same time, interposition cues combined with the color red produce stronger depth percepts and faster perceptual judgments than partial occlusion combined with the colors green or white. This is an important finding because it shows that a strong primary color such as red can override the considerable boundary advantage of configurations with partial occlusion. This effect is not predicted by any theory we know of and highlights the particular status of the color red as a cue to depth. It may be related to the finding that desaturated red targets are found more rapidly than appropriately equated targets of other colors (Lindsey et al., 2010), although such a difference is not always found (Santhi and Reeves, 2004). However, red stimuli are not inevitably seen as nearer in the visual field than stimuli with other colors. Also, the highest probabilities of near, and the fastest perceptual judgments, are produced by red figures combined with a partial occlusion cue or a positional cue presented on a light background. Though this suggests that the color red is a potentially strong candidate as a depth cue with, initially, a strong relative weight, white figures combined with a partial occlusion cue produce stronger depth percepts and faster perceptual judgments than red figures combined with an interposition cue presented on a dark background. This result clarifies that, despite an advantage due to wavelength characteristics 


\section{Dresp-Langley, Reeves}

promoting color stereopsis, red does not always benefit from this advantage, nor does it automatically attract visual attention away from other stimuli in the visual field. It may only do so when certain geometric constraints are met. Abstract expressionists intuitively exploit such color interactions to suggest shapes and figure-ground, as shown in Figure 12.6 (see Color plate XX in the Color plate section).

\section{How does the brain compute figure-ground?}

Finding out about interactions between the different monocular cues to figure and ground, or how they may cooperate or compete in a given configuration, is critical to our understanding of how the brain extracts structural information from a complex image or visual scene. Earlier theories (e.g. Gibson, 1950) emphasized the importance of cooperative interactions, or cue combinations, claiming that information provided by multiple cues would have to be combined to generate unified percepts of shapes and their relative depth within the visual field. Cue combination, which suggests cooperation between cues, would occur when two qualitatively different depth cues contribute to the depth percept at a given location. Conflict, or competition between cues, would occur in situations where an unambiguous cue fails to strengthen an ambiguous one. Although all cues available in a given configuration cooperate in the first instance, cues with little relative weight may then be overridden by a cue with stronger relative weight. Obviously, the brain proceeds to a selection of the most likely signals, which, in a first instance, trigger what is commonly called 'attention'.

\section{Signal selection to trigger visual attention}

It has been suggested that the probability based selection of neural signals may drive perceptual organization by telling the brain to perform the most likely structural analysis of visual input (e.g. Dresp and Langley, 2005). At some stage in this process, attention becomes important as some objects readily attract attention away from others in the visual field. In this process, objects with a stronger luminance contrast may benefit from selection for attention when presented together with objects of a lesser contrast (see again Figure 12.4). Similarly, some colors may attract attention away from others in the visual field (e.g. Dresp and Grossberg, 1999; Yantis and Jones, 1991) and, as explained above, the color red is often readily seen as nearer than others, not only on the basis of color stereopsis, but in many complex visual scenes where multiple factors determine perceptual organization.

Nakayama and Silverman (1986) showed that the visual detection of targets defined by combinations of color and depth in a visual search task is faster than the detection of targets defined by a single attribute. The implications of a correlation between perceived depth in geometric configurations and neural selection for attention were discussed further by Nakayama et al. (1990). If such a correlation exists, the probability that a given object is seen to be nearer than 
another object in the visual field should indeed correlate with the response latencies of near percepts, as suggested by the data summarized here in Figures 12.3a and b. In particular, under normal viewing conditions where luminance contrast, color contrast, stimulus geometry, and visual attention interact, the cue combinations that yield the most likely depth percept should be more salient, thereby promoting selective visual attention. Then, a strong luminance or color cue combined with a strong geometric cue should produce shorter response latencies than a weaker luminance or color cue combined with a weaker geometric cue, as indeed confirmed by the data of Guibal and Dresp (2004).

\section{From contours in the plane to surfaces in depth: Grossberg's FACADE theory}

Grossberg's FACADE (Feature-And-Contour-And-DEpth) model (1997) is a neural model of perceptual organization, with two subsystems (see also Chapter 4 by Breitmeyer in this volume). The Boundary Contour System (BCS) is orientation selective and detects boundaries. The BCS interacts with the Feature Contour System (FCS) of the model, which detects luminance contrasts within image regions defined by boundaries. The combined monocular outputs of BCS and FCS cooperatively feed into a selective BCS-FCS mechanism that groups input from a given location in the visual field. Visual configurations generating a so-called relative boundary advantage yield stronger figure-ground percepts because they generate comparatively stronger signals in the BCS. Functional asymmetries in the processing of brighter versus darker contrasts (Dengler and Nitschke, 1993; De Weert and Spillmann, 1995; Dresp and Langley, 2005; Dresp-Langley and Reeves, 2012; Magnussen and Glad, 1975) are accounted for by the model. The output of BCS-FCS interaction determines whether a stimulus in a given location survives against stimuli in other locations. Bottom-up representations surviving this monocular, competitive stage of the FACADE model are selected and integrated into a binocular form representation. Figureground representations are generated by non-linear, long-range interactions (Spillmann and Werner, 1996), which ensure that changes in lighting conditions or visual adaptation will not hinder object perception. The FACADE predictions are consistent with observations that strong achromatic luminance contrasts, although they may cooperate with any given geometric cue at weaker intensities, generate competitive interactions with other cues, especially with interposition. Partial occlusion survives the competition against a strong luminance cue significantly better than an interposition cue, although both resolve ambiguities about which object is likely to lie in front of which other in the plane (Dresp et al., 2002; Guibal and Dresp, 2004). Why partial occlusion wins against interposition in competition with a strong luminance cue is explained by the relative boundary advantage of partial occlusion cue, with a larger amount of visible boundary information provided by the figure lying in front. This pictorial depth cue was first mentioned by Leonardo da Vinci in his Trattato della Pittura (1651). In scenes representing objects of the same size that do not overlap, the sole cue 


\section{Dresp-Langley, Reeves}

to depth is relative location. FACADE theory predicts that neural expectation signals generated by information of relative spatial location are powerful attractors of visual attention (Grossberg, 1997), and that the perceptual system readily expects objects presented at lower locations in the visual field to be nearer to the human eye because they yield higher probabilities to be immediately relevant. Unlike partial occlusion, a positional cue does not generate a relative boundary advantage, but the figure-ground solution is computed faster than through other geometric cues to depth, which require further structural analyses. Also, several cues combined in a given configuration do not produce simple summation of figure-ground probabilities. This is consistent with cue-mixture models where different probabilistic weights are given to different depth cues, and where the weight of a given cue is conditional upon the relative weight of others (Knill, 2003). Although all cues available in a given configuration cooperate in the first instance, cues with little relative weight may subsequently be overridden by a cue with a stronger relative weight. Unlike the FACADE theory, cue mixture models do not attempt to link figure-ground perception to cortical mechanisms. They use Bayesian principles for computing conditional probability functions, which are the product of the likelihood functions for each given cue. FACADE proposes a biologically more plausible solution where figure-ground representation results from dynamic processes of cooperation and competition between neural ('expectation') signals in cortical pathways. The major postulates from this theory have proven to be consistent with neurophysiological data (e.g. Spillmann and Werner, 1996; von der Heydt and Peterhans, 1989).

\section{Summary/conclusion}

The pioneering work by Lothar Spillmann and his colleagues has inspired original scientific research into visual phenomena such as the Ehrenstein Illusion, the watercolor illusion, and similar configurations. This has deepened our understanding of the processes of perceptual organization which allow the human brain to extract qualia such as brilliancy, figure-ground, and visual object integrity from the most elementary signals of contrast and shape displayed in two-dimensional space. This has opened doors to new and exciting investigations into the effects of color, and their interaction with geometric properties, on our perception of a visual world that is rapidly evolving and increasing in complexity. Modern art, architecture, and conceptual design, where the idea of a colorful world with a freeform geometry now largely dominates over the sober traditional approaches (see also Pinna, 2011), is about to challenge our perceptual system in ways we have not even begun to imagine. Understanding how we adapt to such constantly changing visual environments will no doubt motivate new lines of research aimed at linking perception to the workings of the human brain. 


\section{Acknowledgement}

Financial support from Centre National de la Recherche Scientifique (CNRSPICS05971) is gratefully acknowledged.

\section{References}

Beck, J. (1966) 'Contrast and assimilation in lightness judgments', Perception \& Psychophysics, 1: 342-344.

Brewster, D. (1851) 'Notice of a chromatic stereoscope', Philosophical Magazine, 4th Series, 3: 31 .

Chevreul, M. E. (1839) De la loi du contraste simultané des couleurs et de l'assortiment des objets colorés, English trans. J. Spanton (1854) Laws of Contrast of Color, Milton Keynes: Lightning Source.

Da Vinci, L. (1651) Trattato della Pittura di Leonardo da Vinci. Scritta da Raffaelle du Fresne, Paris, France: Langlois.

Dengler, M. and Nitschke, W. (1993) 'Color stereopsis: A model for depth reversals based on border contrast', Perception \& Psychophysics, 53: 150-156.

Devinck, F., Spillmann, L. and Werner, J. S. (2006) 'Spatial profile of contours inducing long-range color assimilation', Visual Neuroscience, 23: 573-577.

De Weert, C. M. M. and Spillmann, L. (1995) 'Assimilation: Asymmetry between brightness and darkness', Vision Research, 35: 1413-1419.

Dresp, B. and Grossberg, S. (1999) 'Spatial facilitation by color and luminance edges: Boundary, surface, and attention factors', Vision Research, 39: 3431-3443.

Dresp, B. and Fischer, S. (2001) 'Asymmetrical contrast effects induced by luminance and color configurations', Perception \& Psychophysics, 63: 1262-1270.

Dresp, B. and Langley, O. K. (2005) 'Long-range spatial integration across contrast signs: A probabilistic mechanism', Vision Research, 45: 275-284.

Dresp, B., Durand, S. and Grossberg, S. (2002) 'Depth perception from pairs of overlapping cues in pictorial displays', Spatial Vision, 15: 255-276.

Dresp-Langley, B. (2009) 'The Ehrenstein Illusion', Scholarpedia, 4: 53-64.

Dresp-Langley, B. and Reeves, A. (2012) 'Simultaneous contrast and apparent depth from true colors on grey: Chevreul revisited', Seeing o Perceiving, 25(6): 597-618.

Ehrenstein, W. (1941) 'Über Abwandlungen der L. Herrmannschen Helligkeitserscheinung', Zeitschrift für Psychologie, 150: 83-91.

Egusa, H. (1983) 'Effects of brightness, hue, and saturation on perceived depth between adjacent regions in the visual field', Perception, 12: 167-175.

Gerrits, H. J. M. and Vendrik, A. J. (1970) 'Simultaneous contrast, filling-in process, and information processing in mans visual system', Experimental Brain Research, 11: $411-430$.

Gibson, J. J. (1950) The Perception of the Visual World, Boston, MA: Houghton Mifflin.

Grossberg, S. (1997) 'Cortical dynamics of three-dimensional figure-ground perception of two-dimensional figures', Psychological Review, 104: 618-658.

Guibal, C. R. C. and Dresp, B. (2004) 'Interaction of color and geometric cues in depth perception: When does red mean near', Psychological Research, 10: 167-178.

Hamada, J. (1985) 'Asymmetric lightness cancellation in Craik-O'Brien patterns of negative and positive contrast', Biological Cybernetics, 52: 117-122.

Heinemann, E. G. (1955) 'Simultaneous brightness induction as a function of inducing and test-field luminance', Journal of Experimental Psychology, 50: 89-96. 


\section{Dresp-Langley, Reeves}

Katz, D. (1911) 'Die Erscheinungsweisen der Farben und ihre Beeinflussung durch die individuelle Erfahrung', Zeitschrift für Psychologie, 7: 6-31.

Knill, D. (2003) 'Mixture models and the probabilistic structure of depth cues', Vision Research, 43, 831-854.

Lindsey, D. T., Brown, A. M., Reijnen, E., Rich, A. N., Kuzmova, Y. I. and Wolfe, J. M.

(2010) 'Color channels, not color appearance or color categories, guide visual search for desaturated color targets', Psychological Science, 21: 1-21.

Livitz, G., Yazdanbakhsh, A., Eskew, R. T. Jr. and Mingolla, E. (2011) 'Perceiving opponent hues in color induction displays', Seeing \& Perceiving, 24: 1-17.

Magnussen, S. and Glad, A. (1975) 'Brightness and darkness enhancement during flicker: Perceptual correlates of neuronal B- and D- systems in human vision', Experimental Brain Research, 22: 399-413.

Metzger, W. (1930) Gesetze des Sehens, English trans. L. Spillmann (2009) Laws of Seeing Cambridge, MA: MIT Press.

Nakayama, K. and Silverman, G. H. (1986) 'Serial and parallel processing of visual feature conjunctions', Nature, 320: 264-265.

Nakayama, K., Shimojo, S. and Ramachandran, V. S. (1990) 'Transparency, relation to depth, subjective contours, luminance and neon color spreading', Perception, 19: 497-513.

O'Shea, R. P., Blackburn, S. G. and Ono, H. (1994) 'Contrast as a depth cue', Vision Research, 34: 1595-1604.

Oyama, T. and Yamamura, T. (1967) 'The effects of hue and brightness on depth perception in normal and color-blind subjects', Psychologia, 3: 191-194.

Pinna, B. (ed.) (2008) Color, Line and Space: The Neuroscience of Spatio-Chromatic Vision, Leiden, The Netherlands: Brill.

Pinna, B. (2011) 'The organization of shape and color in vision and art', Frontiers in Human Neuroscience, 5: A 104.

Pinna, B. and Reeves, A. (2006) 'Lighting, backlighting and the laws of figurality in the watercolor illusion', Spatial Vision, 19: 341-373.

Pinna, B., Brelstaff, G. and Spillmann, L. (2001) 'Surface color from boundaries: A new “watercolor" illusion', Vision Research, 41: 2669-2676.

Rohaly, A. M. and Wilson, H. R. (1999) 'The effects of contrast on perceived depth and depth discrimination', Vision Research, 39: 9-18.

Ross, H. E. (1967) 'Water, fog, and the size distance invariance hypotheses', British Journal of Psychology, 58: 301-313.

Santhi, N. and Reeves, A. (2004) 'The roles of distractor noise and target certainty in search: A signal detection model', Vision Research, 44: 1235-1256.

Schwartz, B. J. and Sperling, G. (1983) 'Luminance controls the perceived 3D structure of dynamic 2D displays', Bulletin of the Psychonomic Society, 17: 456-458.

Shapley, R. and Reid, R. C. (1985) 'Contrast and assimilation in the perception of brightness', Proceedings of the National Academy of Science, 82: 5983-5986.

Simonet, P. and Campbell, M. C. W. (1990) 'Effect of luminance on the directions of chromatostereopsis and transverse chromatic aberration observed with natural pupils', Ophthalmic \& Physiological Optics, 10: 271-279.

Spillmann, L. (1977) 'Contrast and brightness illusions', in H. Spekreijse and L. H. van der Tweel (eds) Spatial Contrast, Amsterdam, The Netherlands: North-Holland, pp. $45-49$.

Spillmann, L. and Dresp, B. (1995) 'Phenomena of illusory form: Can we bridge between levels of explanation?', Perception, 24: 1333-1364. 
Spillmann, L. and Werner, J. S. (1996) 'Long-range interactions in visual perception', Trends in Neurosciences, 19: 428-434.

Spillmann, L., Fuld, K. and Gerrits, H. J. M. (1976) 'Brightness contrast in the Ehrenstein illusion', Vision Research, 16: 713-719.

Spillmann, L., Fuld, K. and Neumeyer, C. (1984) 'Brightness matching, brightness cancellation, and increment threshold in the Ehrenstein illusion', Perception, 13: 513-520.

Van Tuijl, H. F. J. M. (1975) 'A new visual illusion: Neonlike color spreading and complementary color induction between subjective contours', Acta Psychologica, 39: $441-445$.

Verhoeff, F. H. (1928) 'An optical illusion due to chromatic aberration', American Journal of Ophthalmology, 11: 898-900.

Von der Heydt, R. and Peterhans, E. (1989) 'Ehrenstein and Zöllner illusions in a neuronal theory of contour processing', in J. J. Kulikowski, C. M. Dickinson and I. J. Murray (eds) Seeing contour and color, Oxford, UK: Pergamon Press, pp. 729-734.

Von der Heydt, R. and Pierson, R. (2006) 'Dissociation of color and figure-ground effects in the watercolor illusion', Spatial Vision, 19: 323-340.

Watanabe, T., Nanez, J. F. and Moreno, M. A. (1995) 'Depth release of illusory contour shape in the Ehrenstein grid', Vision Research, 35: 2845-2851.

Wertheimer, M. (1923) Perceived Motion and Figural Organization, English trans. L. Spillmann, M. Wertheimer, K. W. Watkins, S. Lehar and V. Sarris (2012) Cambridge, MA: MIT Press.

Yantis, S. and Jones, E. (1991) 'Mechanisms of attentional selection: Temporally modulated priority tags', Perception \& Psychophysics, 50: 166-178.
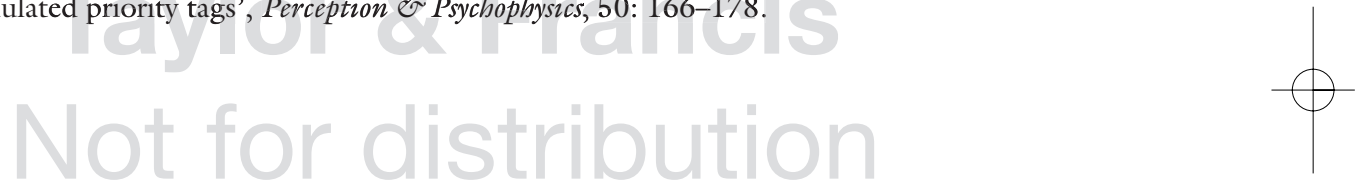